\title{
Thoracoscopic removal of ectopic mediastinal parathyroid adenoma causing hyperparathyroidism: a rare entity
}

\author{
Ezel Erşen, Burcu Kılıç \\ Department of Thoracic Surgery, Cerrahpaşa Medical Faculty, Istanbul University, Istanbul, Turkey
}

Videosurgery Miniinv 2018; 13 (4): 546-550

DOI: https://doi.org/10.5114/wiitm.2018.75896

\begin{abstract}
Ectopic mediastinal parathyroid adenomas are uncommon. Here, we report the successful thoracoscopic removal of a mediastinal parathyroid adenoma from a patient with hypercalcemia. A 58-year-old female patient was referred to our department with persistent hypercalcemia. Serum calcium and phosphorus levels were $13.2 \mathrm{mg} / \mathrm{dl}$ and $2.5 \mathrm{mg} / \mathrm{dl}$ respectively, while the intact parathormone level was $798.9 \mathrm{pg} / \mathrm{ml}$. Parathyroid computed tomography revealed a solid and well-defined mass at the anterior mediastinum close to the distal aorta. Once the contrast was enhanced, our pre-diagnosis was ectopic parathyroid adenoma. The mass was detected at the anterior mediastinum, dissected free from the surrounding tissue and excised. The capsulated mass, which had a diameter of $3 \times 1.5 \times 0.7 \mathrm{~cm}$, was confirmed as parathyroid adenoma histopathologically. Postoperative calcium and parathormone levels decreased dramatically to normal levels. Anterior mediastinal localization of an ectopic parathyroid adenoma is a rare entity. The transsternal approach is the most preferred method, but video-thoracoscopy can be used safely and effectively.
\end{abstract}

Key words: ectopic, parathyroid, video-thoracoscopy, mediastinum.

\section{Introduction}

A hyperfunctioning parathyroid gland is found in an ectopic location in around $6 \%$ to $16 \%$ of patients undergoing parathyroid surgery [1]. Approximately $1 \%$ to $25 \%$ of all ectopic parathyroid glands are present in the mediastinum, and they can be removed through a cervical incision. Two percent of these glands are not accessible through a cervical incision, which requires median sternotomy or thoracotomy for resection [2]. The mediastinoscopic approach has an additional advantage at the upper pole of the thymus as well as the aortopulmonary window, which are the common locations for mediastinal ectopic glands [3]. However, the use of video-assisted thoracic surgery has reduced the need for sternotomy or thoracotomy for lesions occurring in the middle or posterior mediastinal compartment [2, 4]. Robot-as- sisted dissection for mediastinal ectopic parathyroid glands has also been reported to be feasible as it provides better visibility of the recurrent laryngeal nerve and parathyroid glands [5]. In this study, we report the successful thoracoscopic removal of a mediastinal parathyroid adenoma from a patient with hypercalcemia.

\section{Case report}

During her routine follow-up, persistent hypercalcemia was detected in a 58-year-old female patient who had a history of diabetes mellitus and hypertension. The physical examination was normal. Serum calcium and phosphorus levels were $12.61 \mathrm{mg} / \mathrm{dl}$ and $2.08 \mathrm{mg} / \mathrm{dl}$ respectively. She was referred to our department for further evaluation. Her serum calcium and phosphorus levels were $13.2 \mathrm{mg} / \mathrm{dl}$ and $2.5 \mathrm{mg} / \mathrm{dl}$

\section{Address for correspondence}

Ezel Erşen, Department of Thoracic Surgery, Cerrahpaşa Medical Faculty, Istanbul University, 34098 Istanbul, Turkey,

phone: +90 5057311567, e-mail: drezelersen@gmail.com 
respectively, while the intact parathormone level was $798.9 \mathrm{pg} / \mathrm{ml}$. Cervical ultrasonography and thyroid scintigraphy were normal. Parathyroid computed tomography (CT) revealed a solid and well-defined mass at the anterior mediastinum close to the distal aorta. Once the contrast was enhanced, our pre-diagnosis was ectopic parathyroid adenoma (Photo 1). Fine needle aspiration did not yield tumor cells. Thus, we planned the video-thoracoscopic removal of the mass. The patient was positioned in a 30-degree semi-supine position and a roll was placed under the shoulder, while the ipsilateral arm was held abducted over a padded L-screen. Two $10 \mathrm{~mm}$ ports and one $5 \mathrm{~mm}$ port were used. Trocars were positioned at the anterior axillary line in the third and fifth intercostal space and also at the mid-axillary line in the fourth intercostal space. A double-lumen intubation was used and the operation was carried out without $\mathrm{CO}_{2}$ insufflation. The mass was detected at the middle part of the anterior mediastinum (Photo $2 \mathrm{~A}$ ). Intraoperative radio-guided navigation with a gamma probe can be used in smaller lesions for localization, although we did not need to do that since our lesion's diameter was $3 \mathrm{~cm}$.

The ectopic gland was dissected free from the surrounding tissue and excised (Photos $2 \mathrm{~B}$ and 3). The thoracostomy tube was removed, and the patient was discharged on the first post-operative day without any surgical complications. The capsulated mass, which had a diameter of $3 \times 1.5 \times 0.7 \mathrm{~cm}$, was confirmed as a parathyroid adenoma histopathologically. The postoperative calcium levels de-

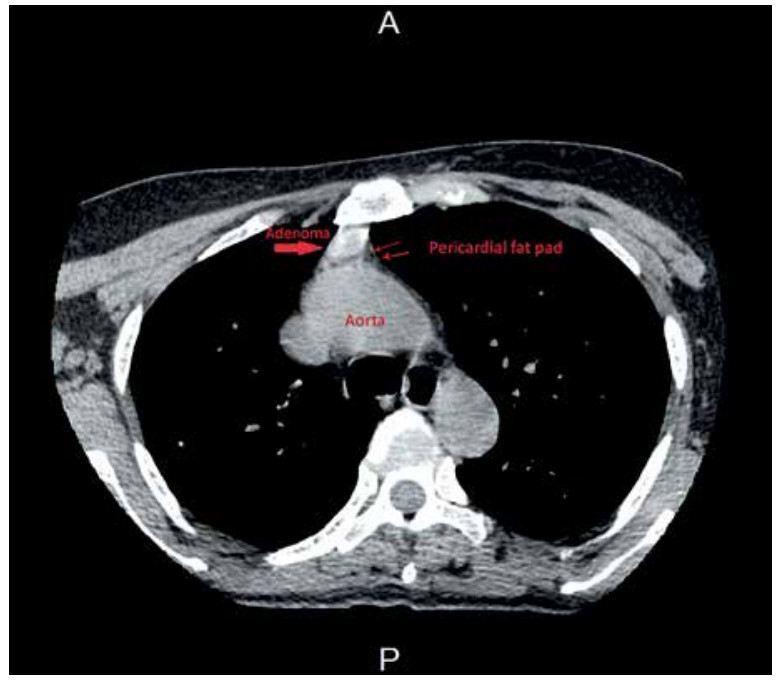

Photo 1. Parathyroid computed tomography revealed a solid, well-defined mass at the anterior mediastinum, close to the distal aorta. The boundary between the mass and pericardial fatty tissue was clearly visible

creased dramatically to the level of $7.5 \mathrm{mg} / \mathrm{dl}$, while the parathormone level decreased to $122.6 \mathrm{pg} / \mathrm{ml}$. The parathormone level was $55.09 \mathrm{pg} / \mathrm{ml}$ and the serum calcium level was $9.55 \mathrm{mg} / \mathrm{dl}$ at the patient's follow-up visit ten months after the surgery.

\section{Discussion}

Hyperparathyroidism is mostly caused by parathyroid adenoma or hyperplasia and it is responsi-
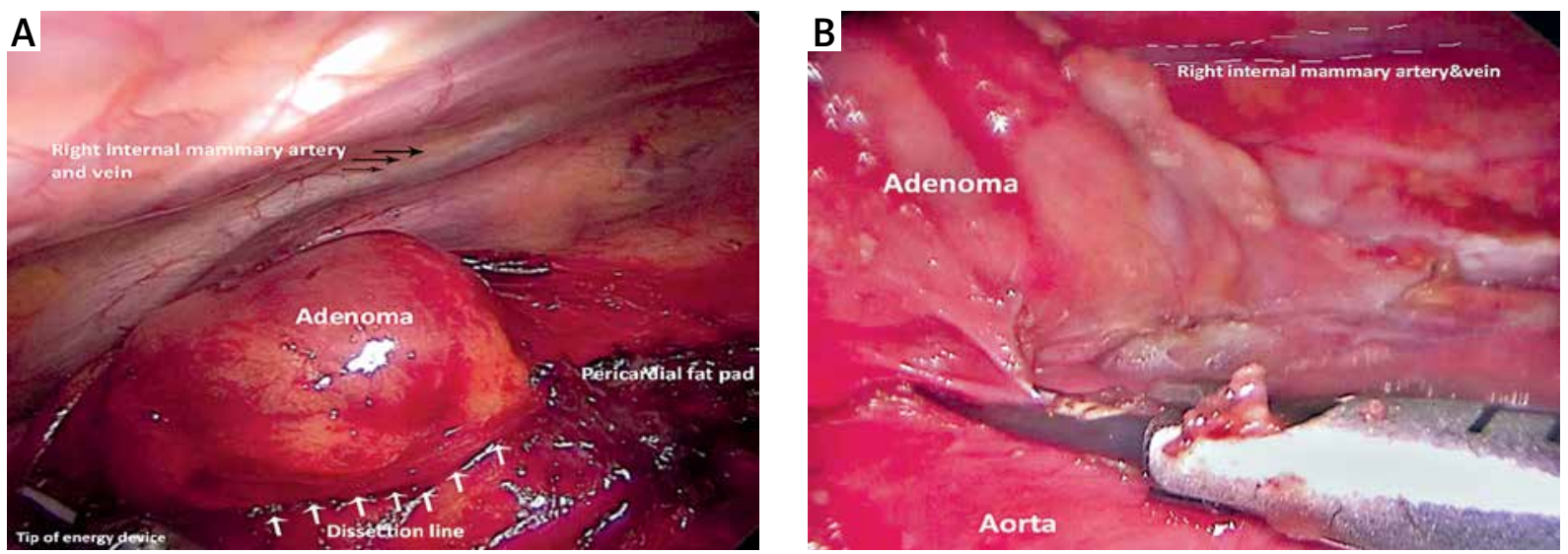

Photo 2. A - Thoracoscopic view of the adenoma at anterior mediastinum, close to the right internal mammary artery, behind the sternum. B - The dissection was started at the lateral border of the vena cava, then the ectopic gland was retracted upwards and dissected free from the anterior aortic wall. The ectopic gland was dissected free from the surrounding tissue using an energy device and excised 


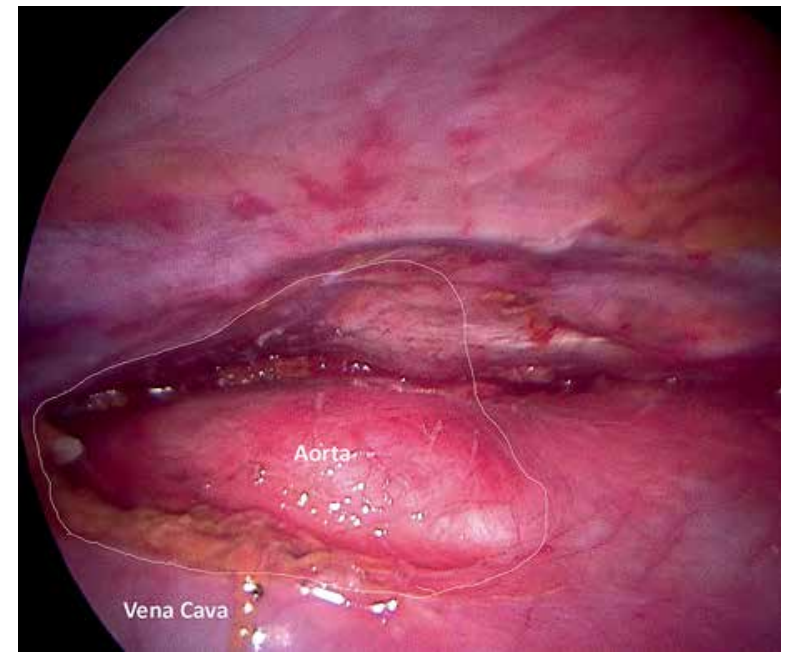

Photo 3. View of mediastinum after excision of the ectopic tissue. The boundary of the dissection is drawn as a white line. An extended resection with secured margins was performed and care was taken not to breach the capsule of the gland

ble for numerous morbidity, such as calciphylaxis, decreased bone density, and dystrophic tissue calcification. Aggressive medical management is suggested for these cases. On the other hand, subtotal parathyroidectomy and total parathyroidectomy with autotransplantation are the surgical strategies to limit the morbidity in refractory cases, for secondary hyperparathyroidism [6].

Ectopic mediastinal parathyroid glands are an important cause of persistent hyperparathyroidism, which can be found in approximately $25 \%$ of patients with primary hyperparathyroidism [7]. Most of the lesions are accessible with a standard cervical approach, although mediastinal exploration is required in around $1 \%$ to $2 \%$ of the patients with ectopic parathyroid $[8,9]$.

It is not routinely recommended to scan the mediastinum for preoperative examination to localize the glands but mediastinal evaluation is always mandatory after a failed cervical exploration, to look for ectopic parathyroid tissue. Mediastinal exploration was performed via a sternotomy for exploring ectopic parathyroids, before the development of modern imaging methods. However, this blind exploration not only had a high failure rate in detecting the ectopic gland but complications associated with sternotomy could be seen in up to $21 \%$ of cases as well [10]. This blind approach is no longer in use and preoperative localization is recommended before any mediastinal exploration.

Historically, standard approaches to ectopic mediastinal parathyroids, which are not accessible via cervical incision, have been sternotomy and anterolateral thoracotomy. However, complication rates associated with these approaches have been reported to be up to $29 \%$ [8, 11].

Pleural effusion, pneumothorax, and pneumonitis (in $21 \%$ of patients) and wound complications such as hematomas, anterior mediastinitis, and sternal dehiscence (in $8 \%$ of patients) were reported by Russell et al. and Conn et al. reported a complication rate of $19 \%$ after sternotomy for resection in patients with ectopic parathyroids [8, 11].

Additionally, these interventions have some disadvantages such as higher morbidity and longer hospital stay. A median hospital stay of 9 days was reported after sternotomy by Doherty et al. [12].

There are some reports in the literature describing other less invasive approaches in patients with mediastinal parathyroids such as anterior mediastinotomy, mediastinoscopy, subxiphoid laparoscopy, angiographic ablation and transcervical anterior mediastinal exploration.

Anterior mediastinotomy was reported to be feasible in selected patients with ectopic adenomas by Schlinkert et al. [13], but in this series the ectopic glands located deep in the mediastinum required additional sternotomy. Wells and Cooper [14] used a special sternal retractor for anterior mediastinal exploration via a transcervical approach, which also ensures removal of the parathyroid glands. Unfortunately, parathyroid glands located in the aorto-pulmonary window or posterior mediastinum cannot be safely removed by this method. Angiographic ablation, as a nonsurgical alternative to standard mediastinal exploration, has also been reported by Doherty et al. [12]. They performed 30 angiographic ablations in 27 patients with ectopic glands. Three patients converted into hypoparathyroidism, while 14 became normocalcemic, and 10 required additional treatment after the procedure. Recurrence developed in 3 patients. The disadvantages of this technique were poor gland localization and a high early failure rate $[15,16]$. Another disadvantage of angio-ablation is that no tissue was available for histopathological examination and for possible future autotransplantation. None of these approaches have been widely accepted as reliable and safe interventions [17]. 
Thoracoscopic excision of a mediastinal parathyroid adenoma was first performed by Prinz et al. in 1994 [9]. In their series, cervical thymectomy was performed on all four patients as part of the first neck examination, but this maneuver did not involve the removal of abnormal mediastinal parathyroid glands. Following this report, some case reports recommended video-assisted thoracoscopic surgery (VATS) as a reliable and safe approach for the resection of ectopic mediastinal parathyroid glands [9, 18]. This minimally invasive approach for mediastinal parathyroidectomy has been reported, mainly as case reports or small series because of the rarity of this type of lesion.

Another article reported the successful removal of a parathyroid adenoma in four cases with ectopic mediastinal parathyroid adenoma. In all of the 4 cases, the exploration through the cervical incision was unsuccessful [17]. In the literature, there are only a few individual case reports of the successful resection of ectopic parathyroid adenomas with the VATS approach [19-23]. Lihara et al. reported 14 patients with hyperparathyroidism, in whom a mediastinal lesion had been localized preoperatively by a sestamibi scan [24]. The thoracoscopic procedure was used successfully in eight patients who were shown preoperatively to have deep-seated (5 anterior, 3 middle) mediastinal lesions. All eight mediastinal lesions localized preoperatively below the level of the aortic arch in the deep mediastinum were successfully removed by thoracoscopy. They also stated that the thoracoscopic approach can be a feasible option for mediastinal re-exploration in patients with recurrence [24].

Computed tomography, magnetic resonance imaging, and nuclear scanning are the modalities currently available for evaluation of the mediastinum. Parathyroid glands which are larger than $1.5 \mathrm{~cm}$ can be detected by CT scan, but it may be difficult to localize smaller parathyroid glands [25]. Magnetic resonance imaging is reported to be more sensitive than $\mathrm{CT}$, but it is not as sensitive as nuclear scanning. The nuclear scan has reported to have a high sensitivity of $86 \%$ for glands greater than $1 \mathrm{~g}$ and $100 \%$ for glands greater than $2 \mathrm{~g}$ [25]. Detection of parathyroid glands as small as $500 \mathrm{mg}$ by nuclear scanning was reported by Ipponsugi et al. [26].

Pre-operative localization is very important for the success of the surgery. A $99 \mathrm{mTc}$ sestamibi parathyroid scan and computed tomography of both the neck and the thorax can easily localize the ectopic parathyroid adenoma. Intraoperative radio-guided navigation with a gamma probe can be used for perioperative localization in smaller lesions [27, 28]. Alesina et al. [18] considered that the use of a gamma probe could be helpful for reducing the operation time. We did not need to do it in our case because our lesion's diameter was $3 \mathrm{~cm}$, and it could be identified easily with direct thoracoscopic exploration.

Video-assisted thoracoscopic surgery offers a unique opportunity with the additional benefits of magnified vision of mediastinal structures. Ectopic parathyroids localized in the mediastinum can be approached and removed by thoracoscopy. The procedure is performed with the patient under general anesthesia with double-lumen intubation to achieve single-lung ventilation. The mediastinal parathyroid can be approached from any side depending on the position of the lesion, as seen in the CT scan. Generally, three trocars are used, and the operation is performed using a standard 10-mm rigid telescope with a $0^{\circ}$ angle. After exploration, the pleura around the ectopic tissue is incised, and the ectopic adenoma is identified, mobilized, and excised with safe margins. The most important issue is not to breach the capsule of the gland. Also an endoscopic bag should be used for the removal. Follow-up in the intensive care unit is generally not required. The chest tube can be removed on the same day or next day and the patient can be discharged.

Thoracoscopic surgery is a safe and feasible option for resection of deep mediastinal parathyroid lesions, even in patients undergoing repeated operations. Video-assisted thoracoscopic surgery can provide excellent access to all mediastinal locations of parathyroids and it has several advantages over the trans-sternal approach such as less pain, shorter operation time and shorter length of hospital stay. Video-assisted thoracoscopic additionally has a superior cosmetic result with a much smaller incision scar. Our patient was discharged on the first post-operative day without any surgical complications.

\section{Conclusions}

The anterior mediastinal localization of an ectopic parathyroid adenoma is a rare entity. Higher levels of parathormone and symptomatic hypercalcemia should urge physicians to search for ectopic locations especially when a suspected parathyroid 
adenoma cannot be found in the neck. The mediastinum should be cautiously investigated since it is the most common location for an ectopic parathyroid adenoma after the neck. The transsternal approach is the most preferred method, although video-thoracoscopy can be used safely and effectively for the treatment of hyperparathyroidism caused by ectopic mediastinal parathyroid adenomas.

\section{Conflict of interest}

The authors declare no conflict of interest.

\section{References}

1. Hu J, Ngiam KY, Parameswaran R. Mediastinal parathyroid adenomas and their surgical implications. Ann R Coll Surg Engl 2015; 97: 259-61.

2. Yutaka Y, Omasa M, Shikuma K, et al. Video-assisted mediastinoscopic resection of ectopic parathyroid adenoma. Asian Cardiovasc Thorac Ann 2012; 20: 731-3.

3. Wei B, Inabnet W, Lee JA, et al. Optimizing the minimally invasive approach to mediastinal parathyroid adenomas. Ann Thorac Surg 2011; 92: 1012-7.

4. Randone B, Costi R, Scatton O, et al. Thoracoscopic removal of mediastinal parathyroid glands: a critical appraisal of an emerging technique. Ann Surg 2010; 251: 717-21.

5. Ismail M, Maza S, Swierzy M, et al. Resection of ectopic mediastinal parathyroid glands with the da Vinci robotic system. Br J Surg 2010; 97: 337-43.

6. Richards ML, Wormuth J, Bingener J, et al. Parathyroidectomy in secondary hyperparathyroidism: is there an optimal operative management? Surgery 2006; 139: 174-80.

7. Smythe WR, Bavaria JE, Hall RA, et al. Thoracoscopic removal of mediastinal parathyroid adenoma. Ann Thorac Surg 1995 59: 236-8.

8. Russell CF, Edis AJ, Scholz DA, et al. Mediastinal parathyroid tumors: experience with 38 tumors requiring mediastinotomy for removal. Ann Surg 1981; 193: 805-9.

9. Prinz RA, Lonchyna V, Carnaille B, et al. Thoracoscopic excision of enlarged mediastinal parathyroid glands. Surgery 1994; 116: 999-1004.

10. Kumar A, Kumar S, Aggarwal S, et al. Thoracoscopy: the preferred method for excision of mediastinal parathyroids. Surg Laparosc Endosc Percutan Tech 2002; 12: 295-300.

11. Conn JM, Goncalves MA, Mansour KA, et al. The mediastinal parathyroid. Am Surg 1991; 37: 62-6.

12. Doherty GM, Doppman JL, Miller DL, et al. Results of a multidisciplinary strategy for management of mediastinal parathyroid adenoma as a cause of persistent primary hyperthyroidism. Ann Surg 1992; 215: 101-6.

13. Schlinkert RT, Whitaker MD, Argueta R. Resection of selected mediastinal parathyroid adenomas through an anterior mediastinotomy. Mayo Clin Proc 1991; 66: 1110-3.

14. Wells SA, Cooper JD. Closed mediastinal exploration in patients with persistent hyperparathyroidism. Ann Surg 1991; 214: 555-61.
15. Heller HJ, Miller GL, Erdman WA, et al. Angiographic ablation of mediastinal parathyroid adenomas: local experience and review of the literature. Am J Med 1994; 97: 529-34.

16. Doppman JL, Brown EM, Brennan MF, et al. Angiographic ablation of parathyroid adenomas. Radiology 1979; 130: 577-82.

17. Chae AW, Perricone A, Brumund KT, et al. Outpatient video-assisted thoracoscopic surgery (VATS) for ectopic mediastinal parathyroid adenoma: a case report and review of the literature. J Laparoendosc Adv Surg Tech 2008; 18: 383-90.

18. Alesina PF, Moka D, Mahlstedt J, et al. Thoracoscopic removal of mediastinal hyperfunctioning parathyroid glands: personal experience and review of the literature. World I Surg 2008; 32: 224-31.

19. Naik D, Jebasingh KF, Ramprasath GBR, et al. Video assisted thoracoscopic surgery (VATS) for excision of an ectopic anterior mediastinal intra-thymic parathyroid adenoma. I Clin Diagn Res 2016; 10: 22-4.

20. Mohamed SE, Li X, Khadra H, et al. Different surgical approaches in parathyroid adenoma resections. Gland Surg 2013; 2: 227-9.

21. Kim YS, Kim J, Shin S. Thoracoscopic removal of ectopic mediastinal parathyroid adenoma. Korean I Thorac Cardiovasc Surg 2014; 47: 317-9.

22. Okagawa T, Hiramatsu Y. Ectopic mediastinal parathyroid tumor resected by video-assisted thoracic surgery with intraoperative methylene blue infusion: report of a case. Kyobu Geka 2014; 67: 255-7.

23. Pecheva M, Mahendran K, Kadlec J, et al. Mediastinal giant parathyroid adenoma - a minimally invasive mediastinal surgical approach for an emergency presentation. Ann Cardiothorac Surg 2016; 5: 70-3.

24. Lihara M, Suzuki R, Kawamata A, et al. Thoracoscopic removal of mediastinal parathyroid lesions: selection of surgical approach and pitfalls of preoperative and intraoperative localization. World I Surg 2012; 36: 1327-34.

25. Medrano C, Hazrelrigg SR, Landreneau RJ, et al. Thoracoscopic resection of ectopic parathyroid glands. Ann Thorac Surg 2000; 69: 221-3.

26. Ipponsugi S, Takamori S, Suga K, et al. Mediastinal parathyroid adenoma detected by $99 \mathrm{mTC}-$ methoxyisobutylisonitrile: report of a case. Surg Today 1997; 27: 80-3.

27. Giron J, Ouhayoun E, Dahan M, et al. Imaging of hyperparathyroidism: US, CT, MRI and MIBI scintigraphy. Eur J Radiol 1996; 21: 167-73.

28. Mehrabibahar M, Mousavi Z, Sadeghi R, et al. Feasibility and safety of minimally invasive radio-guided parathyroidectomy using very low intraoperative dose of Tc-99m MIBI. Int J Surg 2017; 39: 229-33.

Received: 18.01 .2018 , accepted: 3.03.2018. 